\title{
Genome-wide variations analysis of sorghum cultivar Hongyingzi for brewing Moutai liquor
}

Lingbo Zhou, Can Wang, Xu Gao, Yanqing Ding, Bin Cheng, Guobing Zhang, Ning Cao, Yan Xu, Mingbo Shao and Liyi Zhang*

\begin{abstract}
Background: Hongyingzi is a sorghum (Sorghum bicolor L. Moench) cultivar for brewing Moutai liquor. For an overall understanding of the whole genome of Hongyingzi, we performed whole-genome resequencing technology to reveal its comprehensive variations.

Results: Compared with the BTx623 reference genome, we uncovered 1,885,774 single nucleotide polymorphisms (SNPs), 309,381 small fragments insertions and deletions (Indels), 31,966 structural variations (SVs), and 217,273 copy number variations (CNVs). These alterations conferred 29,614 gene variations. It was also predicted that 35 gene variations were related to the multidrug and toxic efflux (MATE) transporter, chalcone synthase (CHS), ATPase isoform 10 (AHA10) transporter, dihydroflavonol-4-reductase (DFR), the laccase 15 (LAC15), flavonol 3'-hydroxylase (F3'H), flavanone 3-hydroxylase (F3H), O-methyltransferase (OMT), flavonoid 3'5' hydroxylase (F3'5'H), UDP-glucose:sterolglucosyltransferase (SGT), flavonol synthase (FLS), and chalcone isomerase (CHI) involved in the tannin synthesis.

Conclusions: These results would provide theoretical supports for the molecular markers developments and gene function studies related to the tannin synthesis, and the genetic improvement of liquor-making sorghum based on the genome editing technology.
\end{abstract}

Keywords: Sorghum, Hongyingzi, Genome-wide variations analysis, Whole-genome resequencing technology

\section{Introduction}

Sorghum [Sorghum bicolor (L.) Moench] is the fifth largest cereal crop in the world after corn (Zea mays L.), wheat (Triticum aestivum L.), rice (Oryza sativa L.), and barley (Hordeum vulgare L.), which is widely distributed in the arid and semi-arid regions of the tropics, and also one of the earliest cultivated cereal crops in China [1]. It has become a model crop for genome research of cereal crops because of its wide adaptability to environment, strong stress resistance, rich resources, and relatively small genome $[2,3]$. According to different purposes,

\footnotetext{
* Correspondence: lyzhang1997@hotmail.com

Institute of Upland Food Crops, Guizhou Academy of Agricultural Sciences, Guiyang 550006, Guizhou, China

(c) The Author(s). 2020 Open Access This article is licensed under a Creative Commons Attribution 4.0 International License, which permits use, sharing, adaptation, distribution and reproduction in any medium or format, as long as you give appropriate credit to the original author(s) and the source, provide a link to the Creative Commons licence, and indicate if changes were made. The images or other third party material in this article are included in the article's Creative Commons licence, unless indicated otherwise in a credit line to the material. If material is not included in the article's Creative Commons licence and your intended use is not permitted by statutory regulation or exceeds the permitted use, you will need to obtain permission directly from the copyright holder. To view a copy of this licence, visit http://creativecommons.org/licenses/by/4.0/. The Creative Commons Public Domain Dedication waiver (http://creativecommons.org/publicdomain/zero/1.0/) applies to the data made available in this article, unless otherwise stated in a credit line to the data. and liquor-making sorghum breeding strategies.
}

sorghum are generally divided into three types, namely sweet sorghum, feed sorghum, and grain sorghum [4]. Sorghum is one of the main raw materials for Moutaiflavor liquor and Luzhou-flavor liquor production due to its high amylopectin content and tannin content $[5,6]$. In recent years, the undiversified main liquor-making sorghum cultivar and its continuous degradation phenomenon has affected the supply of raw materials for liquor-making sorghum and restricted the development of liquor enterprises [7]. Therefore, investigation of liquor-making sorghum genetic resources is a crucial measure for better straight evolution, genetic studies, 
Genetic variation is a kind of variation that can be passed on to offspring due to the changes of genetic material in organisms and leads to the genetic diversity at different levels. There are many types of genetic variation in the genome, from microscopic chromosome inversion to single nucleotide mutation. With the development of genomics, the information of genetic variation that can be studied has become more comprehensive, such as single nucleotide polymorphism (SNP), small fragments insertion and deletion (Indel), structural variation (SV), and copy number variation $(\mathrm{CNV})$ [8-10]. With the rapid development of molecular biology, whole-genome resequencing technology has been applied to genome-wide variations analysis in Arabidopsis, rice, maize, tomato, and other plants $[8,11-13]$. The whole genome sequences of grain sorghum cultivar BTx623 has provided a template for genome-wide variations analysis in sorghum [14], and the first genome-wide variations analysis of sorghum was reported by [15].

Tannin, also known as condensed tannins or proanthocyanidins, is oligomer and polymer of flavan-3-ols. It is widespread throughout the plant kingdom, with diverse biological and biochemical functions, such as protection against predation from herbivorous animals and pathogenic attack from bacteria and fungi [16]. Tannin is found in seeds of sorghum with a pigmented testa layer and it has been shown high antioxidant and dietary fiber levels, and to decrease protein digestibility and feed efficiency in humans and animals [17]. High tannin content is the main reason why sorghum has become a raw material for brewing famous liquor and tannin content is closely related to the brewing flavor, such as sorghum cultivar with between 1 and $2 \%$ of tannin content is raw material for brewing Moutai-flavor liquor [18, 19]. Previous studies have mapped some gene loci associated with tannin content of sorghum. The Tan1 gene (Sb04g031730) was cloned, which code a WD40 protein and control the tannin biosynthesis [16]. Two gene loci linked to tannin content were also found [20]. One was named as Sb01g001230, coding glutathione-S-transferase, and another was named as Sb02g006390, coding bHLH transcription factor and was isotopic with gene $B_{2}$ for color seed coat.

Hongyingzi, a sorghum cultivar used for brewing Moutai liquor containing $83.40 \%$ total starch, $96.27 \%$ amylopectin/total starch ratio, and $1.61 \%$ tannin. However, the genome information of Hongyingzi is not fully understood. Here, we used whole-genome resequencing technology to resequence Hongyingzi genome to identify patterns of sequence polymorphism and structural variation in comparison with the published BTx623 genome. This effort identified a large quantity of SNPs, Indels, SVs, and CNVs. Comparison of these variation data defined potential genome regions and metabolic pathways associated with tannin synthesis. Such knowledge are useful for genetic improvement and tailor-designed breeding of liquor-making sorghum.

\section{Materials and methods Plant materials}

Hongyingzi was used in this study, which approved by the Guizhou Crop Cultivar Approval Committee (Guiyang, Guizhou Province, China) in 2008, is a medium maturity sorghum cultivar used for brewing Moutai liquor and developed by Renhuai Fengyuan Organic Sorghum Breeding Center at Guizhou, China in 2008 [21]. Seeds of Hongyingzi were sterilized by soaking in $0.1 \%$ mercury dichloride for $15 \mathrm{~min}$, and then rinsed with distilled water for ten times. Next, seeds were placed in a germination box lined with three layers of filter paper and added 15 $\mathrm{mL}$ distilled water. The germination box was placed in the RXZ-1000B artificial climate box for cultivating 10 days as following parameters settings, day/night temperature is $28^{\circ} \mathrm{C} / 25^{\circ} \mathrm{C}$, light/dark time is $12 \mathrm{~h} / 12 \mathrm{~h}$, humidity is $85 \%$, and light intensity is $340 \mu \mathrm{mol} \mathrm{m} \mathrm{m}^{-2} \mathrm{~s}^{-1}$.

\section{DNA isolation and resequencing}

The 10-day-old healthy seedlings were harvested for DNA extraction using the CTAB buffer method [22]. The DNA purity was determined by $0.8 \%$ agarose gel $100 \mathrm{~V}$ electrophoresis for $40 \mathrm{~min}$ and DNA concentration was determined by Qubit $^{\circ} 2.0$ fluorescent meter (Invitrogen, Carlsbad, USA). Following quality assessment, the genomic DNA was randomly broken into 350 bp fragments by Covaris ultrasonic crushing apparatus and DNA fragments were end repaired, added ployA tail, added sequencing connector, purification, and PCR amplification to complete the establishment of the library. The constructed library was used to paired-end PE150 sequencing on Illumina HiSeq 4000 sequencing platform by Beijing Novogene technology co., LTD (Beijing, China).

\section{Filtering reads and mapping reads}

The original image data generated by the sequencing machine were converted into sequence data via base calling (Illumina pipeline CASAVA v1.8.2) and then subjected to quality control procedure to remove unusable reads according to following criteria: the reads contain the Illumina library construction adapters, the reads contain more than $10 \%$ unknown bases ( $\mathrm{N}$ bases), and one end of the read contain more than $50 \%$ of low quality bases (sequencing quality value $\leq 5$ ). After filtration, sequencing reads were aligned to the BTx623 reference genome using BWA v0.7.8 [23] with the parameters as 'mem -t 4 -k 32 -M'. Subsequent processing, including duplicate removal was performed using SAMtools v0.1.19 [24] with the parameter as 'rmdup'. The BTx623 reference genome sequences were downloaded from the 
https://phytozome.jgi.doe.gov/pz/portal.html\#!info?alias= Org_SbicolorRio_er.

\section{SNP and Indel analyses}

SNP and Indel detections were performed using SAMtools v0.1.19 with the parameters as 'mpileup -m 2 -F $0.002-d 1000$ '. Based on the filtering and mapping results, the clean data were assembled and estimated using a Bayesian model. The performance of SNP detection was based on the following criteria: average quality of the novel allele $>20$, adjacent SNPs were separated by $\geq 5 \mathrm{bp}$, and at least four reads supported the genotype. For Indel detecting, mapped reads met the pair-end requirements and contained alignment gaps in one end. We mapped the paired-end reads to the reference sequence by allowing up to $50 \mathrm{bp}$ gaps. Then, gaps supported by at least three non-redundant paired-end reads were extracted. A potential Indel was identified when the number of ungapped reads were $<2$. The annotations of SNP and Indel were performed using ANNOVAR software [25], and the information on genes with SNPs and Indels were downloaded from the Sorghum bicolor Genome Database [26].

\section{SV and CNV analyses}

$\mathrm{SV}$ and $\mathrm{CNV}$ detections were performed using BreakDancer v1.4.4 [27] and CNVnator v0.3 [28] with the parameter as '-call 100', respectively. According to the principle of paired-end sequencing, one read of a pairedend should be aligned to the forward sequence, while the other read of a paired-end should be aligned to the reverse sequence. The distance between the two aligned positions should be in accordance with the insert size. Thus, the alignment of the two paired reads to the genome is regarded to be of normal direction and appropriate spanning. If the direction or spanning of the alignments of the two paired reads is different from expectation, the region might have a structural variation. The abnormal paired-end alignments were analyzed by clustering and compared with the types of SVs as previously defined. The SVs were detected in the same manner, with support from at least two abnormal paired-end read. After determination of SVs, the total number of CNVs was counted. The annotations of SV and CNV were performed using ANNOVAR software, and the information on genes with SVs and CNVs were also downloaded from the Sorghum bicolor Genome Database.

\section{Gene variation analysis}

Using the BTx623 gene set as the reference, genes with non-synonymous SNPs and Indels in coding regions identified in the Hongyingzi were selected as the candidate gene set. These genes were aligned to the NCBI using Blast2go JavaScript. Gene Ontology (GO) numbers
Table 1 Summary of resequencing data of Hongyingzi

\begin{tabular}{lcccccc}
\hline $\begin{array}{l}\text { Raw base } \\
(\mathrm{Gb})\end{array}$ & $\begin{array}{l}\text { Clean base } \\
(\mathrm{Gb})\end{array}$ & $\begin{array}{l}\text { Effect } \\
\text { rate (\%) }\end{array}$ & $\begin{array}{l}\text { Error } \\
\text { rate (\%) }\end{array}$ & $\begin{array}{l}\text { Q20 } \\
(\%)\end{array}$ & $\begin{array}{l}\text { Q30 } \\
(\%)\end{array}$ & $\begin{array}{l}\text { GC } \\
\text { content } \\
(\%)\end{array}$ \\
\hline 45.84 & 45.79 & 99.82 & 0.03 & 97.55 & 93.10 & 44.30 \\
\hline
\end{tabular}

were downloaded from the Sorghum bicolor Genome Database and imported to the WEGO database [29] for clustering analysis. Genes that were involved in the tannin synthesis were selected and mapped to Kyoto Encyclopedia of Genes and Genomes (KEGG) [30] sorghum pathway data and were examined for whether they are enriched in particular pathways based on the hypergeometric distribution test. Fisher's exact test was used to identify pathways significantly enriched $(P<0.1)$ with tannin synthesis associated genes.

\section{Results}

\section{Genome-wide identification of genetic variations in Hongyingzi}

The whole genome of Hongyingzi was resequenced using Illumina Genome Analyser sequencing technology. The genome size of the BTx623 reference genome is 732.15 $\mathrm{Mb}$. Resequencing yielded $45.84 \mathrm{~Gb}$ of raw data, which comprised $45.79 \mathrm{~Gb}$ of high quality clean data (Table 1 ). There was a high sequencing quality (Q20 $\geq 97.55 \%$, $\mathrm{Q} 30 \geq 93.10$ ) and the GC content was $44.30 \%$. The results showed that the 297,504,853 reads obtained with Hongyingzi were mapped to the BTx623 reference genome, with an effective depth of $56.10 \mathrm{X}$ coverage, 95.94\% of coverage at least one base, and $94.17 \%$ of coverage at least four bases (Table 2). With these reads and the information from the BTx623 reference genome, large quantities of SNPs, Indels, SVs, and CNVs were identified (Fig. 1). Compared with the BTx623 reference genome, we finally found 1,885,774 SNPs, 309,381 Indels, 31,966 SVs, and 217,273 CNVs in Hongyingzi. These variations were distributed relatively evenly across 10 chromosomes of sorghum and had the most distribution on chromosome 1 as well as a few were distributed on extra chromosomes.

\section{SNPs in the Hongyingzi genome}

A total of $1,885,774$ SNPs were identified in the Hongyingzi genome, including 1,230,508 transitions and 655, 266 transversions. Besides, there were 1,401,089 homozygous SNPs and 484,685 heterozygous SNPs (Fig. S1 in Additional file 1 ), and the het rate was $0.066 \%$. As shown

Table 2 Sequence alignment of Hongyingzi to BTx623

\begin{tabular}{lccccc}
\hline $\begin{array}{l}\text { Mapped } \\
\text { reads }\end{array}$ & Total reads & $\begin{array}{l}\text { Mapping } \\
\text { rate (\%) }\end{array}$ & $\begin{array}{l}\text { Average } \\
\text { depth }(X)\end{array}$ & $\begin{array}{l}\text { Coverage } \\
\text { at least } \\
1 \text { base } \\
(\%)\end{array}$ & $\begin{array}{l}\text { Coverage } \\
\text { at least } \\
4 \text { bases } \\
(\%)\end{array}$ \\
\hline $297,504,853$ & $305,064,750$ & 97.52 & 56.10 & 95.94 & 94.17 \\
\hline
\end{tabular}


by annotations of SNPs detected in Hongyingzi (Table 3), there were 1,515,993 SNPs mutation in intergenic, 89, 326 SNPs in $1 \mathrm{~kb}$ of upstream, 75,170 SNPs in $1 \mathrm{~kb}$ of downstream, and 6344 SNPs mutated in both $1 \mathrm{~kb}$ of upstream and another $1 \mathrm{~kb}$ of downstream. We found that 76,528 SNPs were mutated in exonic regions, including 38,176 synonymous SNPs, 37,774 nonsynonymous SNPs, 453 SNPs related to gain of stop codons, and 125 SNPs related to loss of stop codons. We also found that there were 122,211 SNPs mutation in intronic regions and 202 SNPs in splicing sites. Besides, the proportion of $\mathrm{C}: \mathrm{G}>\mathrm{T}: \mathrm{A}$ type was observed to be the highest (Fig. S2 in Additional file 1).

\section{Indels in the Hongyingzi genome}

A total of 309,381 Indels containing 149,071 insertions and 160,310 deletions, was uncovered in the Hongyingzi genome. These Indels also included 309,361 homozygous and 20 heterozygous Indels (Fig. S3 in Additional file 2 ), and the het rate was $0.0065 \%$. Annotation analysis (Table 3) showed that there were 190,165, 38, 198, 28,361, and 2779 Indels mutated in intergenic, $1 \mathrm{~kb}$ of upstream, $1 \mathrm{~kb}$ of downstream, and both $1 \mathrm{~kb}$ of upstream and another $1 \mathrm{~kb}$ of downstream, respectively. We found that 9375 Indels were mutated in exonic regions, in which 103 Indels were related to gain of stop codons, 22 Indels were related to loss of stop codons, 1354 insertions and 1476 deletions might lead to frameshift, and 3219 insertions and 3201 deletions might lead to non-frameshift. We also found 40,223 Indels were mutated in intronic regions and 189 Indels did in splicing sites. Besides, the proportion of $1 \mathrm{bp}$ and $3 \mathrm{bp}$ Indels (Fig. S4 in Additional file 2) were observed to be the highest in whole genome and coding regions, respectively.

\section{SVs in the Hongyingzi genome}

A total of 31,966 SVs were identified in the Hongyingzi genome, including 70 insertions, 15,975 deletions, 1948 inversions, 4938 intrachromosomal translocations, and

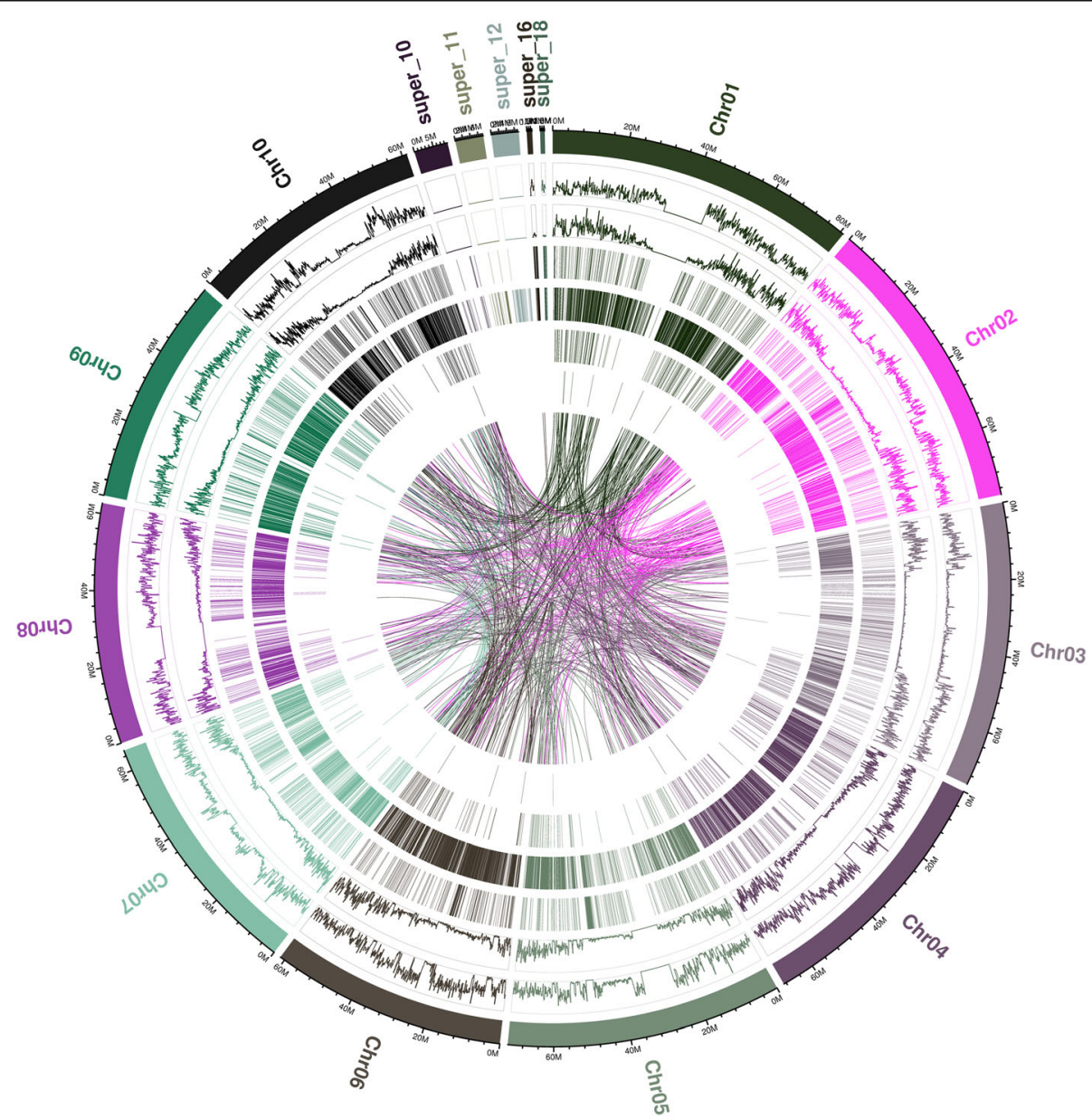

Fig. 1 Genome-wide landscape of genetic variation in Hongyingzi. Cycles from outside to inside indicate chromosome, single nucleotide polymorphism (SNP), small fragments insertion and deletion (Indel), copy number variation (CNV) duplication and deletion, structural variation (SV) insertion, SV deletion, SV invertion, and SV intrachromosomal translocation (ITX) and SV interchromosomal translocation (CTX) 
Table 3 Annotations of single nucleotide polymorphisms (SNPs), small fragments insertions and deletions (Indels), structural variations (SVs), and copy number variations (CNVs) detected in Hongyingzi

\begin{tabular}{|c|c|c|c|c|c|}
\hline Category & Numbers of SNPs & Numbers of Indels & Numbers of SVs & Numbers of CNVs & Region \\
\hline Intergenic & $1,515,993$ & 190,165 & 9661 & 17,082 & \\
\hline $1 \mathrm{~kb}$ of upstream & 89,326 & 38,198 & 1915 & 985 & \\
\hline $1 \mathrm{~kb}$ of downstream & 75,170 & 28,361 & 1460 & 789 & \\
\hline Upstream/downstream & 6344 & 2779 & 176 & 96 & \\
\hline Gain of stop codons & 453 & 103 & & & Coding regions \\
\hline Loss of stop codons & 125 & 22 & & & Coding regions \\
\hline Synonymous & 38,176 & & & & Coding regions \\
\hline Non-synonymous & 37,774 & & & & Coding regions \\
\hline Frameshift (insertions) & & 1354 & & & Coding regions \\
\hline Frameshift (deletions) & & 1476 & & & Coding regions \\
\hline Non-frameshift (insertions) & & 3219 & & & Coding regions \\
\hline Non-frameshift (deletions) & & 3201 & & & Coding regions \\
\hline Exonic & & & 3657 & 1822 & \\
\hline Intronic & 122,211 & 40,223 & 1119 & 496 & \\
\hline Splicing sites & 202 & 189 & 5 & & \\
\hline
\end{tabular}

9035 interchromosomal translocations (Fig. S5 in Additional file 3). As shown by annotations of SVs detected in Hongyingzi (Table 3), there were 9661 SVs mutation in intergenic, 1915 in $1 \mathrm{~kb}$ of upstream, 1460 in $1 \mathrm{~kb}$ of downstream, and 176 in both $1 \mathrm{~kb}$ of upstream and another $1 \mathrm{~kb}$ of downstream. We also found that there were 3657 SVs mutation in exonic regions, 1119 in intronic regions, and 5 in splicing sites.

\section{CNVs in the Hongyingzi genome}

A total of $217,273 \mathrm{CNVs}$ including 4966 duplications and 16,307 deletions was uncovered in the Hongyingzi genome (Fig. S6 in Additional file 3). Annotation analysis (Table 3) showed that there were 17,082, 985, 789, and $96 \mathrm{CNVs}$ mutated in intergenic, $1 \mathrm{~kb}$ of upstream, 1 $\mathrm{kb}$ of downstream, and both $1 \mathrm{~kb}$ of upstream and another $1 \mathrm{~kb}$ of downstream, respectively. We also found that there were $1822 \mathrm{CNVs}$ and $496 \mathrm{CNVs}$ mutated in exonic and intronic regions, respectively.

\section{Functional clustering of gene variations}

Compared to the BTx623 reference genome, 29,614 gene variations were identified in the Hongyingzi genome (Fig. 2). Of which, 14,028, 25,166 and 3948 was caused by SNPs, Indels, and SVs, respectively. GO annotation showed that SNPs and Indels were distributed among different gene ontologies (Fig. 3). In cellular component ontology, the cell and cell part contained the majority of gene variations with $19.06 \%$ SNPs and $23.01 \%$ Indels. Extracellular matrix contained a lower rate of variation. In molecular function ontology, binding and catalytic activity had a higher rate of variation. Binding included
40.77 and $37.56 \%$ of variation in SNPs and Indels, while catalytic activity did 34.01 and $31.67 \%$ of variation in SNPs and Indels. In biological process ontology, metabolic process and cellular process had a high rate of variation. Metabolic process term included 39.00 and $36.07 \%$ of variation in SNPs and Indels, while cellular process did 39.05 and $35.99 \%$ of variation in SNPs and Indels. In KEGG annotation, 141 gene variations caused by SNPs (Fig. 4a) involved in the ubiquitin mediated proteolysis, while 1756 caused by Indels (Fig. 4b) involved in the metabolic pathways. These variations may affect the distinguishing traits between Hongyingzi and BTx623.

\section{Gene variations involved in tannin synthesis}

Compared to the BTx623 reference genome, we found that 35 gene variations were related to the tannin synthesis in the Hongyingzi genome (Table 4). Of which, 7 genes did in the multidrug and toxic efflux (MATE) transporter, 7 involved in the chalcone synthase (CHS), 4 did in the ATPase isoform 10 (AHA10) transporter, 4 did in the dihydroflavonol-4-reductase (DFR), 3 did in the laccase 15 (LAC15), 2 did in the flavonol 3'-hydroxylase (F3' $\mathrm{H}), 2$ did in the flavanone 3-hydroxylase (F3H), 2 did in the $O$-methyltransferase (OMT), 1 did in the flavonoid $3^{\prime} 5^{\prime}$ hydroxylase $\left(\mathrm{F}^{\prime}{ }^{\prime}{ }^{\prime} \mathrm{H}\right), 1$ did in the UDPglucose:sterol-glucosyltransferase (SGT), 1 did in the flavonol synthase (FLS), and 1 did in the chalcone isomerase (CHI). We selected 11 genes with only 1 bp nonsynonymous SNP variation in coding regions to DNA sequence alignment (Fig. 5), and found that these 11 genes altered the coding amino acids. 


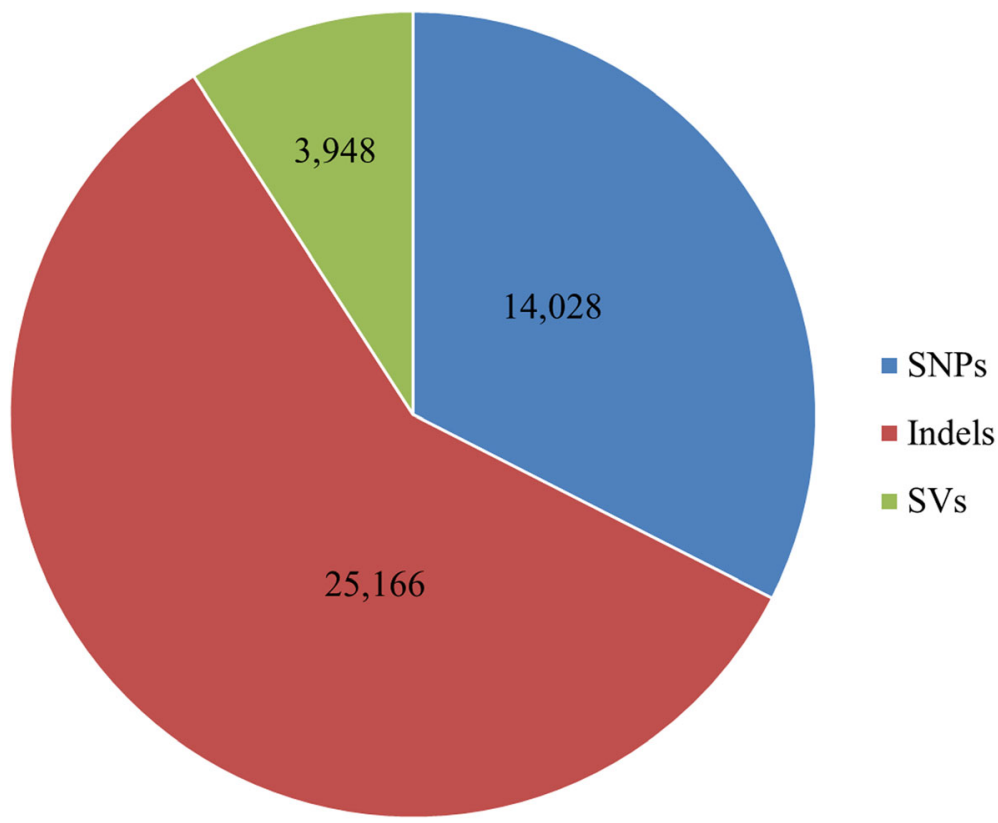

Fig. 2 Summary of genes with three types of variations, single nucleotide polymorphism (SNPs), small fragments insertions and deletions (Indels), and structural variations (SVs). The blue pie indicates SNPs, the red pie indicates Indels, and the green pie indicates SVs

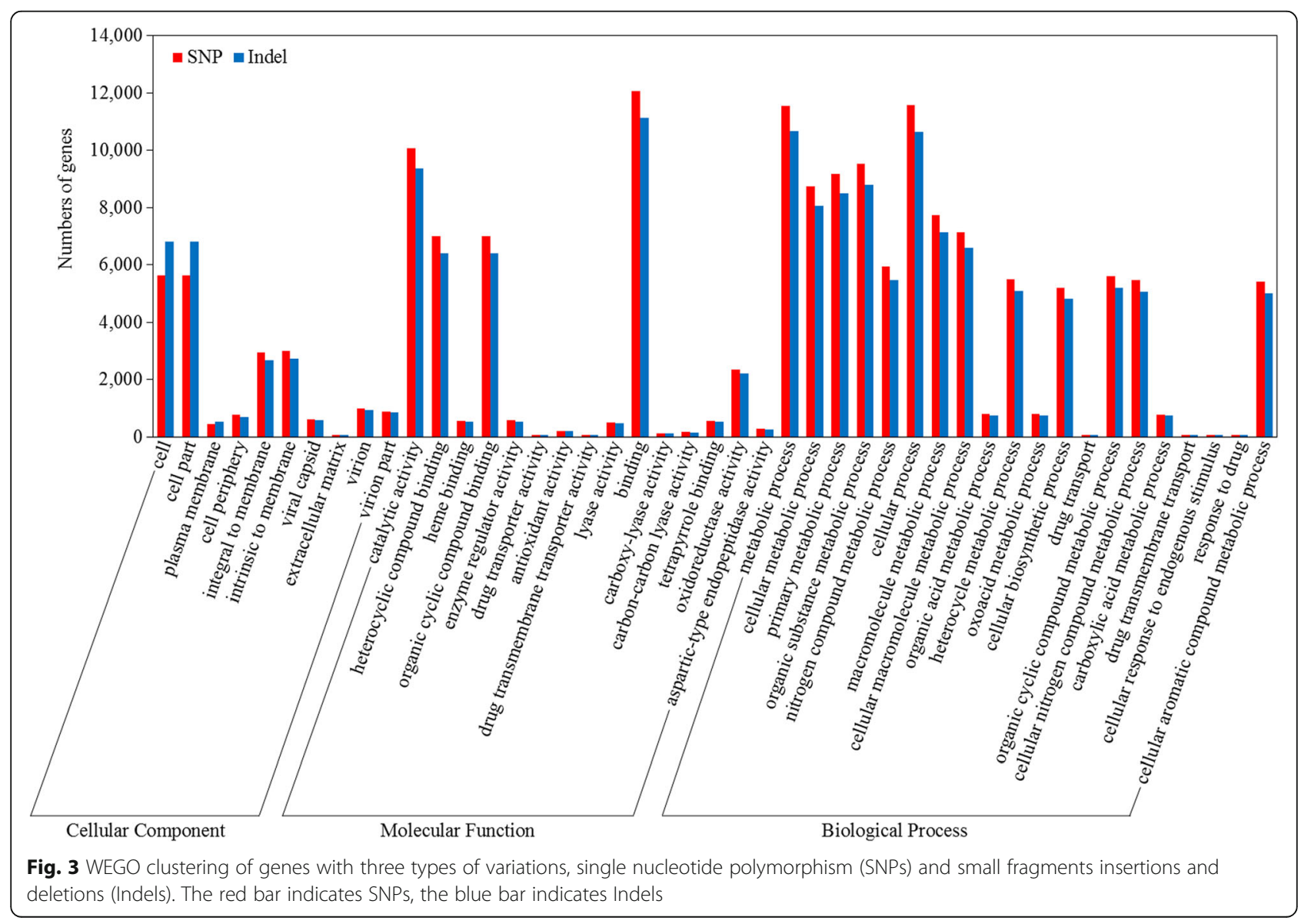



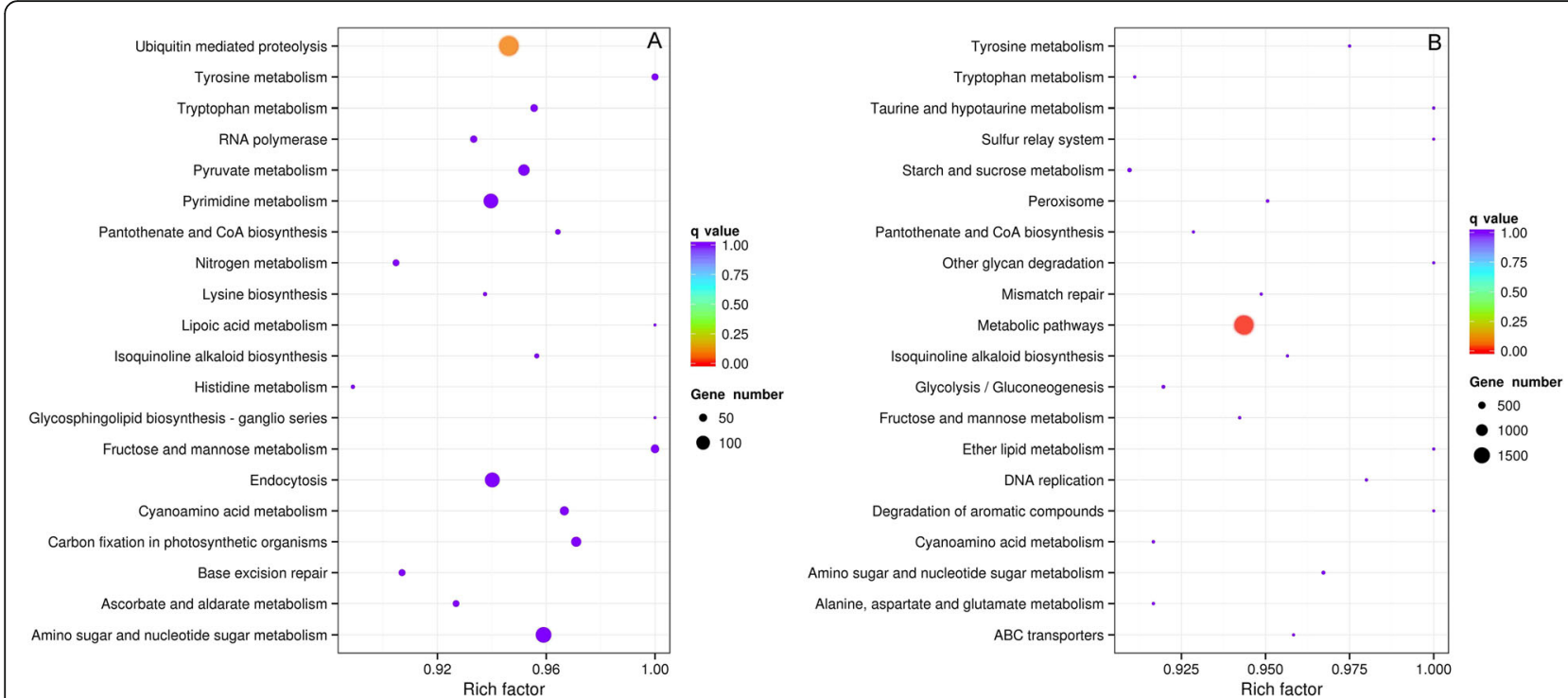

Fig. 4 Classification of gene variations compared with Kyoto Encyclopedia of Genes and Genomes (KEGG) database. a: Top 20 significantly enriched pathways with single nucleotide polymorphism (SNPs) variations. $\mathbf{b}$ : Top 20 significantly enriched pathways with small fragments insertions and deletions (Indels) variations. Gene number: number of target genes in each term or pathway. Rich factor: the ratio of the number of target genes divided by the number of all the gene in each term or pathway

\section{Discussion}

The rapid development of high-throughput sequencing technologies and bioinformatic tools makes it possible to understand the genetic variation and diversity of sorghum at the whole genome level, and then are useful for genetic improvement and tailor-designed breeding of sorghum [14, 15, 31]. In this study, we used wholegenome resequencing technology to analyze the genetic variation in Hongyingzi, which is a sorghum cultivar for brewing Moutai liquor. The results showed that a lot of genome sequences were different between Hongyingzi and BTx623, and more than 2 million SNPs and Indels, along with large numbers of SVs and CNVs were identified. This is the first report on the genome-wide variations analysis in liquor-making sorghum, which will be valuable for further genotype-phenotype studies and for molecular marker assisted breeding of liquor-making sorghum.

In this study, the proportion of SNPs identified as in intronic regions was $6.48 \%$. Compared to Arabidopsis [32], the intronic regions of sorghum genes harbor more SNPs, which might be related the increased size of the introns; the average intron size of sorghum is $444 \mathrm{bp}$, but for Arabidopsis it is $168 \mathrm{bp}$ [15]. A large number of SNPs was identified to alter in 202 splicing sites, 453 gain of stop codons, and 125 loss of stop codons. These alterations could lead to open reading frames extension, functional gene expression failure, or intron size increase $[8,11,33]$. Besides, the proportion of $3 \mathrm{bp}$ Indels was observed to be the highest in coding regions. This might be due to the loss or increase of three bases results in the deletion or addition of a single amino acid without disrupting the overall reading frame [34], which could be a protection means to avoid the drastic changes of the genetic coding information, and then reduce damage to organisms due to natural variation. In addition, Indels with no multiples of $3 \mathrm{bp}$ were rare in coding regions but relatively common in non-coding regions, because most of frameshift mutations is harmful to sorghum survival [15]. Compared to the BTx623 reference genome, a large number of SVs and CNVs was presented in the Hongyingzi genome, and the annotations of SVs and CNVs were similar to that of SNPs and Indels.

Compared to the BTx623 reference genome, there were 29,614 gene variations in the Hongyingzi genome and Indels accounted for most of the gene variations. However, previous studies reported that SNPs accounted for most of the gene variations in Arabidopsis [35] and sorghum [15]. There are two possible reasons: 1) different materials used in different research, 2) limitations of early sequencing technology. Studies of SVs and CNVs in sorghum lag behind those in other plants. Recent studies in maize showed it potentially contributed to the heterosis during domestication and disease responses $[36,37]$. Thus, we should focused on non-synonymous SNPs and Indels in coding regions for subsequent analysis of mutative genes. In our study, GO annotation showed that the mutative genes were equal distribution in different GO term. This indicates that SNPs and Indels may share similar survival and distribution patterns, although the origins and scales may different for affected genome segments. 
Table 4 Variation genes involved in tannin synthesis

\begin{tabular}{|c|c|c|c|c|}
\hline Gene name & Chromosome & Annotation & Variation type & Variation information \\
\hline Sobic.001G012600 & 1 & SBMATE & Non-synonymous SNP & $1,175,307 \mathrm{bp}, \mathrm{C} / \mathrm{A}$ \\
\hline \multirow[t]{2}{*}{ Sobic.001G185400 } & 1 & SBMATE & Non-synonymous SNP & $\begin{array}{l}15,851,598 \mathrm{bp}, \mathrm{G} / \mathrm{A} ; 15,851,639 \mathrm{bp}, \mathrm{C} / \mathrm{T} ; 15,851,643 \mathrm{bp}, \mathrm{G} / \mathrm{C} \\
15,851,644 \mathrm{bp}, \mathrm{G} / \mathrm{C} ; 15,857,015 \mathrm{bp}, \mathrm{G} / \mathrm{A}\end{array}$ \\
\hline & & & Non-frameshift insertion & $15,851,633 \mathrm{bp},-/ \mathrm{GGT}$ \\
\hline Sobic.001G185500 & 1 & SbMATE & Non-frameshift insertion & $15,867,230 \mathrm{bp},-/ \mathrm{GCACGG}$ \\
\hline \multirow[t]{2}{*}{ Sobic.001G185600 } & 1 & SbMATE & Non-synonymous SNP & 15,877,514 bp, G/T; 158,77,530 bp, T/A; 15,879,898 bp, T/G \\
\hline & & & Non-frameshift deletion & 15,880,626 bp, ACCGGCGCC/- \\
\hline Sobic.004G349550 & 4 & SbMATE & Non-frameshift insertion & $67,834,717 \mathrm{bp},-/ \mathrm{GCTGCT}$ \\
\hline Sobic.004G349600 & 4 & SbMATE & Non-synonymous SNP & $67,848,132$ bp, C/G; 67,848,135 bp, C/G; 67,848,250 bp, T/G \\
\hline Sobic.007G165500 & 7 & SbMATE & Non-synonymous SNP & $60,025,990 \mathrm{bp}, \mathrm{C} / \mathrm{A}$ \\
\hline Sobic.001G360800 & 1 & $S b F 3^{\prime} 5^{\prime} H$ & Non-synonymous SNP & $65,069,116 \mathrm{bp}, \mathrm{T} / \mathrm{C}$ \\
\hline Sobic.001G543900 & 1 & SbAHA10 & Non-synonymous SNP & $80,740,375 \mathrm{bp}, \mathrm{C} / \mathrm{G}$ \\
\hline Sobic.003G436400 & 3 & SbAHA10 & Non-frameshift insertion & $73,733,903 \mathrm{bp},-/ \mathrm{CCG}$ \\
\hline \multirow[t]{5}{*}{ Sobic.010G063700 } & 10 & SbAHATO & Non-synonymous SNP & $\begin{array}{l}\text { 5,033,142 bp, C/A; 5,033,614 bp, C/T; 5,033,877 bp, } \\
\text { C/A; 5,034,044 bp, C/T; 5,034,053 bp, G/C }\end{array}$ \\
\hline & & & Frameshift insertion & $\begin{array}{l}5,032,581 \mathrm{bp},-/ \mathrm{GC} ; 5,032,758 \mathrm{bp},-/ \mathrm{GAGC} ; \\
5,033,017 \mathrm{bp},-/ \mathrm{ATCT}\end{array}$ \\
\hline & & & Non-frameshift deletion & 5,032,669 bp, GTGCTGTTC/- \\
\hline & & & Non-frameshift insertion & $\begin{array}{l}5,033,742 \mathrm{bp},-/ \mathrm{GGG} \\
5,034,105 \mathrm{bp},-/ \mathrm{TTCCAC}\end{array}$ \\
\hline & & & Gain of stop codons & $5,034,223 \mathrm{bp}$, -/CTATTTCA \\
\hline Sobic.010G207800 & 10 & SbAHA10 & Non-synonymous SNP & $55,088,876 \mathrm{bp}, \mathrm{A} / \mathrm{C}$ \\
\hline Sobic.002G117500 & 2 & SbSGT & Non-synonymous SNP & $14,508,960 \mathrm{bp}, \mathrm{C} / \mathrm{A}$ \\
\hline Sobic.002G310500 & 2 & $\mathrm{SbCHS}$ & Non-synonymous SNP & $68,442,264 \mathrm{bp}, \mathrm{A} / \mathrm{C} ; 68,442,283 \mathrm{bp}, \mathrm{G} / \mathrm{A}$ \\
\hline Sobic.004G179000 & 4 & $\mathrm{SbCHS}$ & Non-synonymous SNP & $53,190,344 \mathrm{bp}, \mathrm{C} / \mathrm{T}$ \\
\hline Sobic.005G135600 & 5 & $\mathrm{SbCHS}$ & Non-synonymous SNP & $\begin{array}{l}\text { 58,503,342 bp, T/A; 58,503,472 bp, T/C; 58,503,507 bp, } \\
\text { G/A; 58,503,555 bp, C/G }\end{array}$ \\
\hline Sobic.005G136200 & 5 & $\mathrm{SbCHS}$ & Non-synonymous SNP & $58,859,286 \mathrm{bp}, \mathrm{C} / \mathrm{G}$ \\
\hline Sobic.005G136300 & 5 & $\mathrm{SbCHS}$ & Non-synonymous SNP & $58,881,162 \mathrm{bp}, \mathrm{G} / \mathrm{A}$ \\
\hline Sobic.005G137100 & 5 & $\mathrm{SbCHS}$ & Non-synonymous SNP & $58,943,632 \mathrm{bp}, \mathrm{C} / \mathrm{T}$ \\
\hline \multirow[t]{2}{*}{ Sobic.008G036800 } & 8 & $\mathrm{SbCHS}$ & Non-synonymous SNP & $3,477,776 \mathrm{bp}, \mathrm{G} / \mathrm{A}$ \\
\hline & & & Non-frameshift deletion & $3,477,795 \mathrm{bp}, \mathrm{ACG} /-$ \\
\hline Sobic.003G230900 & 3 & $S b D F R$ & Non-synonymous SNP & $57,029,960 \mathrm{bp}, \mathrm{C} / \mathrm{T}$ \\
\hline Sobic.003G231000 & 3 & $S b D F R$ & Non-frameshift deletion & $57,041,941$ bp, CTGGGA/- \\
\hline Sobic.004G050200 & 4 & SbDFR & Non-frameshift deletion & 4,052,019 bp, AAC/- \\
\hline Sobic.009G043800 & 9 & SbDFR & Non-synonymous SNP & $\begin{array}{l}\text { 4,149,752 bp, T/C; 4,149,842 bp, G/A; 4,149,896 bp, } \\
\mathrm{G} / \mathrm{T} ; 4,149,998 \mathrm{bp}, \mathrm{T} / \mathrm{C} ; 4,150,031 \mathrm{bp}, \mathrm{C} / \mathrm{G}\end{array}$ \\
\hline \multirow[t]{2}{*}{ Sobic.004G200900 } & 4 & $S b F 3^{\prime} H$ & Non-synonymous SNP & $55,234,140 \mathrm{bp}, \mathrm{T} / \mathrm{G}$ \\
\hline & & & Non-frameshift deletion & $55,233,739 \mathrm{bp}, \mathrm{CGGGAA}-$ \\
\hline Sobic.009G162500 & 9 & $S b F 3^{\prime} H$ & Non-synonymous SNP & $51,944,205 \mathrm{bp}, \mathrm{A} / \mathrm{G} ; 51,948,174 \mathrm{bp}, \mathrm{C} / \mathrm{G}$ \\
\hline Sobic.004G236000 & 4 & SbLAC15 & Non-synonymous SNP & $\begin{array}{l}\text { 58,382,355 bp, G/A; 58,382,419 bp, G/A; 58,383,602 bp } \\
\text { A/G; 28,383,682 bp, G/T }\end{array}$ \\
\hline \multirow[t]{2}{*}{ Sobic.004G236100 } & 4 & SbLAC15 & Non-synonymous SNP & $58,391,947 \mathrm{bp}, \mathrm{C} / \mathrm{T}$ \\
\hline & & & Frameshift deletion & 58,392,294 bp, CTAC/- \\
\hline \multirow[t]{3}{*}{ Sobic.005G156700 } & 5 & SbLAC15 & Non-synonymous SNP & $62,814,031 \mathrm{bp}, \mathrm{G} / \mathrm{A} ; 62,814,043 \mathrm{bp}, \mathrm{A} / \mathrm{G} ; 62,814,250 \mathrm{bp} . \mathrm{C} / \mathrm{G}$ \\
\hline & & & Non-frameshift deletion & 62,816,156 bp, CGTCAACGT/- \\
\hline & & & Frameshift deletion & $62,813,716$ bp, C/-; 62,813,926 bp, A/-;62,814,183 bp, C/- \\
\hline
\end{tabular}


Table 4 Variation genes involved in tannin synthesis (Continued)

\begin{tabular}{lllll}
\hline Gene name & Chromosome & Annotation & Variation type & Variation information \\
\hline Sobic.004G310100 & 4 & & Frameshift insertion & $62,813,832 \mathrm{bp},-/ \mathrm{A} ; 62,814,474 \mathrm{bp},-/ T \mathrm{~A}$ \\
Sobic.006G253900 & 6 & SbFLS & Non-synonymous SNP & $64,699,203 \mathrm{bp}, \mathrm{G} / \mathrm{A}$ \\
Sobic.006G254000 & 6 & SbF3H & Non-synonymous SNP & $59,157,048 \mathrm{bp}, \mathrm{A} / \mathrm{T} ; 59,157,274 \mathrm{bp}, \mathrm{C} / \mathrm{T} ; 59,158,255 \mathrm{bp}, \mathrm{T} / \mathrm{A}$ \\
Sobic.007G047300 & 7 & $\mathrm{SbF} 3 \mathrm{H}$ & Non-synonymous SNP & $59,160,879 \mathrm{bp}, \mathrm{A} / \mathrm{C} ; 59,161,461 \mathrm{bp}, \mathrm{G} / \mathrm{A}$ \\
Sobic.010G052200 & 10 & SbOMT & Non-synonymous SNP & $4,721,737 \mathrm{bp}, \mathrm{G} / \mathrm{C} ; 4,721,966 \mathrm{bp}, \mathrm{C} / \mathrm{T} ; 4,724,116 \mathrm{bp}, \mathrm{T} / \mathrm{C}$ \\
Sobic.008G030100 & 8 & SbOMT & Non-synonymous SNP & $4,072,017 \mathrm{bp}, \mathrm{C} / \mathrm{G}$ \\
\hline
\end{tabular}

Tannin, also known as condensed tannin or proanthocyanidins, is oligomers and polymers of flavan-3-ols [16, 17]. Sorghum has been the raw material for making famous liquor because of its grains containing tannin, and contributed special taste to Moutai-flavor liquor $[18,19]$. Compared to the BTx623 reference genome, 35 gene variations were related to the tannin synthesis in the Hongyingzi genome. The genes involved in the MATE transporter, CHS, AHA10 transporter, DFR, LAC15, F3' H, F3H, OMT, F3' 5 ' H, SGT, FLS, and CHI. Of these 35 gene variations, 3 MATE, 6 CHS, 2 AHA10, 2 DFR, 1 LAC15, 1 F3H, 2 F3'H, 2 OMT, 1 F3'5'H, 1 FLS and 1 $\mathrm{CHI}$ had only non-synonymous SNP variation, 2 MATE and 1 AHA10 had only non-frameshift insertion variation, 2 DFR had only non-frameshift deletion variation,
1 MATE had both non-synonymous SNP and nonframeshift insertion variations, 1 MATE, 1 CHS and 1 F3' $\mathrm{H}$ had both non-synonymous SNP and nonframeshift deletion variations, 1 AHA10 had both nonsynonymous SNP, frameshift insertionand, nonframeshift deletion, non-frameshift insertion and gain of stop codons variations, 1 LAC15 had both nonsynonymous SNP and frameshift deletion variations, and 1 LAC15 had both non-synonymous SNP, nonframeshift deletion, and frameshift insertion variations. There were no other reports about these variation and whether those genes only presence in Hongyingzi should for further studies. Furtherly, we selected 11 genes with only 1 bp non-synonymous SNP variation in coding regions to DNA sequence alignment, and found that

\footnotetext{
A $\begin{gathered}\text { BTx } 623 \\ \text { Hongyingzi }\end{gathered}$ CGTCCTCAACGGCGTGCAGCCCGTGCTCTCCGGCGTCGCCGTGGGCTGCGGTTGGCAGGCGTTCGTGG CGTCCTCAACGGCGTGCAGCCCGTGCTCTCCGGCGTCGCCGTGGGCTGCGGTTGGCAGGCGTTCGTGGA GTACGTCAACG : GTACGTCAACG : 80

B $\begin{gathered}\text { BTx623 } \\ \text { Hongyingzi }\end{gathered}$ ATGCCCGGCGTCGCCATCTACCTTCGCCTCCTTATATCCCCGTCCGCCTCCGACCCTTCTTCTACCTCCATCGATCTCGA : 80

C BTx623 : : ATGCCCGGCGTCGCCATCTACCTTCGCCTCCTTATATCC CGTCCGCCTCCGACCCTTCTTCTACCTCCATCGATCTCGA : 80

C $\stackrel{\text { BTx623 }}{\text { Hongyingzi }}$ ACGGAGCGCCTGTCCCTATGGAAAGCCGACCTGGCGGACGAAGGCAGCTTCGACGACGCCATCAGGGGCTGCACCGGCGT : 80

$\mathrm{D}_{\text {Hongyingzi }}^{\text {BTx623 }}$ ACGGAGCGCCTGTCCCTATGGAAAGCCGACCTGGCGGACGAAGGCAGCTTCGACGACGTCATCAGGGGCTGCACCGGCGT : 80

E $\underset{\text { Hongyingzi }}{\text { BTx623 }}$

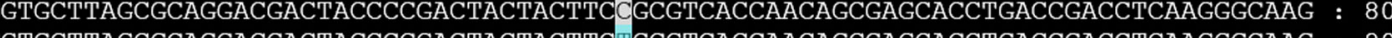

F $\underset{\text { Hongyingzi }}{\text { BTx623 }}$ GGCCTCCACGAGGGCGCCATGGCGGAGGCGTTCGGCGGCGACGGCCTGGTGTTCCTGCACAAGGTCAACTTCTACCCGCC : 80 GGCCTCCACGAGAGCGCCATGGCGGAGGCGTTCGGCGGCGACGGCCTGGTGTTCCTGCACAAGGTCAACTTCTACCCGCC : 80 ATGGCCGCCGCGACTGTGACCGTCGAGGAGGTGAGGAAGGCGCAGCGCGCGACGGGCCCGGCCACCGTGCTGGCGATCGG : 80

$\mathrm{G}_{\text {Hongyingzi }}^{\text {BTx623 }}$ ATGGCCGGCGCGACTGTGACCGTCGAGGAGGTGAGGAAGGCGCAGCGCGCGACGGGCCCGGCCACCGTGCTGGCGATCGG : 80

$\mathrm{H} \underset{\text { Hongyingzi }}{\mathrm{BT} 623}$ TCAAGGAGTGGGGGCAGCCGAAATCCAAGATCACCCACCTCGTCTTCTGCACCACCTCCGGCGTCGACAT TCAAGGAGTGGGGGCAGCCGAAATCCAAGATCACCCACCTCGTCTTCTGCACCACCTCCGGCGTCGACAT ATGCAGGGGCGACGAGACGGGCAGCGTGCGGACGCGGACACCGACACCCCACGCACCCCGTGTTGCATCGCGAGCGCGAG : 80 ATGCAGGGGCGACGAGACGGGCAGCGTGCGGACGCGGACACCGACACCCCACGCACCCA

I $\underset{\text { Hongyingzi }}{\text { BTx623 : }}$ AGCCTGGCTCCGTCATCACTTTCCATTTTCCGGCGACGCCGGGCCCCGCCGACATCACCTTCGTCACGGAGGGGAAAGCG : 80 AGCCTGGCTCCGTCATCACTTTCCATTTTCCGGCGACGCCGGGCCCCGCCGA ATCACCTTCGTCACGGAGGGGAAAGCG : 80

$\mathrm{J} \underset{\text { Hongyingzi }}{\mathrm{BTx} 623}:$ TCGGGATCGTCACGCTGCTCTTCATCAACTCCACGATCAGCTTCATCGAGGAGAACAACGCCGGCAATGCCGCCGCCGCG : 80 TCGGGATCGTCACGCTGCTCTTCATCAACTCCACGATCAGCTTCATCGAGGAGACCAACGCCGGCAATGCCGCCGCCGCG : 80

$\mathrm{K} \underset{\text { Hongyingzi }}{\mathrm{BT} 623}:$ ATGGCCACCACGGCGACCGAGGCGGCCAAGGCCGCGCCGGCGGAGCAGGCCAACGGCAACGCCAACGGCGAGCAGAAGAC : 80 ATGGCCACCACGGCGACCGAGGCGGCAAGGCCGCGCCGGCGGAGCAGGCCAACGGCAACGCCAACGGCGAGCAGAAGAC : 80

Fig. 5 DNA sequence alignments of 11 genes that had only 1 bp non-synonymous SNP variation in coding regions. a: Sobic.001G012600. b: Sobic.001G543900. c: Sobic.003G230900. d: Sobic.004G179000. e: Sobic.004G310100. f: Sobic.005G136200. g: Sobic.005G136300. h: Sobic.007G165500. i: Sobic.008G030100. j: Sobic.010G207800. k: Sobic.010G052200
} 
Sobic.001G012600 had an alteration from arginine to serine, Sobic.001G543900 had an alteration from proline to alanine, Sobic.003G230900 had an alteration from proline to serine, Sobic.004G179000 had an alteration from arginine to cysteine, Sobic.004G310100 had an alteration from glycine to serine, Sobic.005G136200 had an alteration from alanine to glycine, Sobic.005G136300 had an alteration from alanine to threonine, Sobic.007G165500 had an alteration from proline to glutamine, Sobic.008G030100 had an alteration from threonine to arginine, Sobic.010G207800 had an alteration from isoleucine to threonine, and Sobic.010G052200 had an alteration from alanine to glycine. Whether those alterations affect the tannin synthesis in Hongyingzi should for further studies. Expectantly, these variations would provide theoretical supports for the molecular markers developments and gene cloning, and the genetic improvement of liquor-making sorghum based on the genome editing technology.

\section{Conclusions}

This is the first report of genome-wide variations analysis in liquor-making sorghum. High-density SNP, Indel, SV, and CNV reported here will be a valuable resource for future gene-phenotype studies and the molecular breeding of liquor-making sorghum. Gene variations involved in tannin synthesis reported here will provide theoretical basis for marker developing and gene cloning.

\section{Supplementary information}

Supplementary information accompanies this paper at https://doi.org/10. 1186/s41065-020-00130-4.

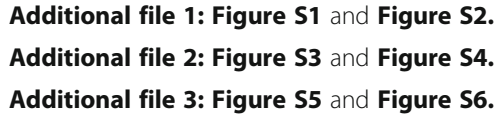

\section{Abbreviations}

AHA10: ATPase isoform 10: CHI: Chalcone isomerase: CHS: Chalcone synthase; CNV: Copy number variation; DFR: Dihydroflavonol-4-reductase; F3H: Flavanone 3-hydroxylase; F3'H: Flavonol 3'-hydroxylase; F3'5'H: Flavonoid 3'5' hydroxylase; FLS: Flavonol synthase; GO: Gene Ontology; Indel: Small fragments insertion and deletion; KEGG: Kyoto Encyclopedia of Genes and Genomes; LAC15: Laccase 15; MATE: Multidrug and toxic efflux; OMT: Omethyltransferase; SNP: Single nucleotide polymorphism; SV: Structural variation; SGT: UDP-glucose:sterol-glucosyltransferase

\section{Acknowledgements}

We thank the bioinformatics team at Beijing Novogene technology Co., LTD for assistance in the analysis of sequencing data.

\section{Authors' contributions}

Zhang LY and Zhou LB conceived the idea of this study. Gao X, Ding YQ, Yu $Y$, and Cheng B collected samples. Wang C, Zhang GB, Cao N, and Shao MB collected samples. Zhang LY, Wang C and Zhou LB wrote the manuscript. All authors read and approved the final manuscript.

\section{Funding}

This research was supported by the National Natural Science Foundation of China (grant number 31660400), Science and Technology Program of Guizhou Province (grant numbers QKHJC20201Y103 and QKHFQ20184005), Special Funds for Guizhou Academy of Agricultural Sciences (grant numbers QNKYZZZY202007 and QNKYYZX2014034), Special Funds for the Central Government Guides Local Science and Technology Development (grant number QKZYD20184003), and Talent Base for Germplasm Resources Utilization and Innovation of Characteristic Plant in Guizhou (grant number RCJD2018-14).

\section{Availability of data and materials}

The raw sequence data in the fastq format from this study were deposited in the NCBI Short Read Archive (SRA) under the accession number SRR11355755. All data produced by the study are disclosed in the manuscript and the additional files.

Ethics approval and consent to participate

This study was approved by the Ethics Committee of Institute of Upland Food Crops, Guizhou Academy of Agricultural Sciences.

\section{Consent for publication}

Not applicable.

\section{Competing interests}

The authors declare that they have no competing interests.

Received: 17 December 2019 Accepted: 14 April 2020

Published online: 14 May 2020

\section{References}

1. Zou GH, Zhai GW, Feng Q, Yan S, Wang AH, Zhao Q, Shao JF, Zhang ZP, Zou JQ, Han B, Tao YZ. Identification of QTLs for eight agronomically important traits using an ultra-high-density map based on SNPs generated from high-throughput sequencing in sorghum under contrasting photoperiods. J Exp Bot. 2012;63(15):5451-62.

2. Nagaraja Reddy R, Madhusudhana R, Murali Mohan S, Chakravarthi DVN, Mehtre SP, Seetharama N, Patil JV. Mapping QTL For grain yield and other agronomic traits in post-rainy sorghum [Sorghum bicolor (L.) Moench]. Theor Appl Genet 2013;126(8):1921-1939.

3. Boyles RE, Pfeiffer BK, Cooper EA, Rauh BL, Zielinski KJ, Myers MT, Brenton Z, Rooney WL. Kresovich, S. Genetic dissection of sorghum grain quality traits using diverse and segregating populations. Theor Appl Genet 2017;130(4): 697-716.

4. Wang C, Zhou LB, Zhang GB, Xu Y, Zhang LY, Gao X, Gao J, Jiang N, Shao $M B$. Drought resistance identification and drought resistance indices screening of liquor-making waxy sorghum resources at adult plant stage. Sci Agric Sin. 2017;50(8):1388-402.

5. Ni XL, Zhao GL, Liu TP, Long WJ, Hu JL, Ding GX. Genetic diversity analysis of glutinous sorghum germplasm resources based on SSR markers. Agric Sci Technol. 2016;17(3):499-504.

6. Wang C, Zhou LB, Zhang GB, Xu Y, Zhang LY, Gao X, Gao J, Jiang N, Shao MB. Optimal fertilization for high yield and good quality of waxy sorghum (Sorghum bicolor L. Moench). Field Crops Res. 2017;203:1-7.

7. Gao X, Zhou LB, Zhang GB, Shao MB, Zhang LY. Genetic diversity and population structure of grain sorghum germplasm resources based on SSR marker. Guizhou Agric Sci. 2016;44(9):13-9.

8. Cheng ZX, Lin JC, Lin TX, Xu M, Huang ZW, Yang ZJ, Huang XY, Zheng JG. Genome-wide analysis of radiation-induced mutations in rice (Oryza sativa L. ssp. indica). Mol BioSyst. 2014;10(4):795-805.

9. Vergara IA, Tarailo-Graovac M, Frech C, Wang J, Qin ZZ, Zhang T, She R, Chu JSC, Wang K, Chen NS. Genome-wide variations in a natural isolate of the nematode Caenorhabditis elegans. BMC Genomics. 2014;15:255.

10. Shao XH, Hu CH, Sheng O, Bi FC, Deng GM, Yang QS, Yi GJ. 2018. Genomewide variations of triploid banana (AAA group) 'grand Nain' by wholegenome resequencing. Plant Physiol J. 2018;54(4):581-93.

11. Lai JS, Li RQ, Xu X, Jin WW, Xu ML, Zhao HN, Xiang ZK, Song WB, Ying $K$, Zhang $M$, Jiao $M$, Jiao YP, Ni PX, Zhang JG, Li D, Guo XS, Ye KX, Jian M, Wang B, Zheng HS, Liang HQ, Zhang XQ, Wang SC, Chen SJ, Li JS, Fu Y, Springer NM, Yang HM, Wang J, Dai JR, Schnable PS, Wang J. Genome-wide 
patterns of genetic variation among elite maize inbred lines. Nat Genet. 2010:42:1027-30.

12. Long $\mathrm{Q}$, Rabanal FA, Meng DZ, Huber CD, Farlow A, Platzer A, Zhang QR, Vilhjálmsson BJ, Korte A, Nizhynska V, Voronin V, Korte P, Sedman L, Mandáková T, Lysak MA, Seren Ü, Hellmann I, Nordborg M. Massive genomic variation and strong selection in Arabidopsis thaliana lines from Sweden. Nat Genet. 2013;45:884-90.

13. Ercolano MR, Sacco A, Ferriello F, D'Alessandro R, Tononi P, Traini A, Barone A, Zago E, Chiusano ML, Delledonne M, Frusciante L. Patchwork sequencing of tomato San Marzano and Vesuviano varieties highlights genome-wide variations. BMC Genomics. 2014;15:138.

14. Paterson AH, Bowers JE, Bruggmann R, Dubchak I, Grimwood J, Gundlach H, Haberer G, Hellsten U, Mittros T, Pollakov A, Schmutz J, Spannagl M, Tang HB, Wang XY, Wicker T, Bharyi AK, Chapman J, Feltus FA, Gowik U, Grigoriev IV, Lyons E, Maher CA, Martis M, Narechania A, Otillar RP, Penning BW, Salamov AA, Wang Y, Zhang LF, Carpita NC, Freeling M, Gingle AR, Thomas Hash C, Keller B, Klein P, Kresovich S, Mccann MC, Ming R, Peterson DG, Rahman M, Ware D, Westhoff P, KFX M, Messing J, Rokhsar DS. The Sorghum bicolor genome and the diversification of grasses. Nature. 2009;457:551-6.

15. Zheng LY, Guo XS, He B, Sun $L$, Peng Y, Dong SS, Liu TF, Jiang SY, Ramachandran S, Liu CM, Jing HC. Genome-wide patterns of genetic variation in sweet and grain sorghum (Sorghum bicolor). Genome Biol. 2011; 12:R114.

16. Wu YY, Li XR, Xiang WW, Zhu CS, Lin ZW, Wu Y, Li JR, Pandravada S, Ridder DD, Bai GH, Wang ML, Trick HN, Bean SR, Tuinstra MR, Tesso TT, Yu JM. Presence of tannins in sorghum grains is conditioned by different natural alleles of Tannin1. Proc Natl Acad Sci U S A. 2012;109(26):10281-6.

17. Zhang CL, Li YF, Zhao WJ, Zhao L, Wang C, Liang D, Zhou FP. Molecular genetic basis for biotechnological improvement of grain quality characteristics in sorghum. Plant Physiol J. 2015;51(5):610-6.

18. Xiong XQ, Chen RX, Yang F, Liu ZS, Zhou YF. Inspection and identification of germplasm resources of wine-making sorghum in Guizhou. J Mt Agric Biol. 2003;22(2):117-21.

19. Bai CM, Wang CY, Wang P, Zhu ZX, Lu XC. QTLs analysis of tannin content and color of grain in sorghum. J Plant Genet Res. 2017;18(5):860-6.

20. Morris GP, Rhodes DH, Brenton Z, Ramu P, Thayil VM, Deshpande S, Hash CT, Acharya C, Mitchell SE, Buckler ES, Yu JM, Kresovich S. Dissecting genome-wide association signals for loss-of-function phenotypes in sorghum flavonoid pigmentation traits. Genes Genom Genet. 2013;3(11): 2085-94.

21. Huang WP, Chen Q. High yield cultivation technique of new sorghum cultivar Hongyingzi. Agric Technol Serv. 2010;27(4):427 442.

22. Zhang LG, Cheng ZJ, Qin RZ, Qiu Y, Wang JL, Cui XK, Gu LF, Zhang X, Guo $X P$, Wang D, Jiang L, Wu CY, Wang HY, Cao XF, Wan JM. Identification and characterization of an epi-allele of FIE1 reveals a regulatory linkage between two epigenetic marks in rice. Plant Cell. 2012;24(11):4407-21.

23. Li H, Durbin R. Fast and accurate short read alignment with burrowswheeler transform. Bioinformatics. 2009;25(14):1754-60.

24. Li H, Handsaker B, Wysoker A, Fennell T, Ruan J, Homer N, Marth G, Abecasis $G$, Durbin R. The sequence alignment/map format and SAMtools. Bioinformatics. 2009:25(16):2078-9.

25. Wang K, Li MY, Hakonarson H. ANNOVAR: functional annotation of genetic variants from high-throughput sequencing data. Nucleic Acids Res. 2010; 38(16):e164.

26. Sorghum bicolor genome. http://www.plantgdb.org/SbGDB/.

27. Chen K, Wallis JW, McLellan MD, Larson DE, Kalicki JM, Pohl CS, McGrath SD, Wendl MC, Zhang QY, Locke DP, Shi XQ, Fulton RS, Ley TJ, Wilson RK, Ding K, Mardis ER. BreakDancer: an algorithm for high-resolution mapping of genomic structural variation. Nat Methods. 2009:6:677-81.

28. Abyzov A, Urban AE, Snyder M, Gerstein M. CNVnator: an approach to discover, genotype, and characterize typical and atypical CNVs from family and population genome sequencing. Genome Res. 2011;21:974-84.

29. WEGO Database. http://wego.genomics.org.cn/cgi-bin/wego/index.pl.

30. KEGG: Kyoto Encyclopedia of Genes and Genomes. http//www.genome.jp/keg.

31. Reuter JA, Spacek DV, Snyder MP. High-throughput sequencing technologies. Mol Cell. 2015;58(4):586-97.

32. Clark RM, Schweikert G, Toomajian C, Ossowski S, Zeller G, Shinn P, Warthmann N, Hu TT, Fu G, Hinds DA, Chen HM, Frazer KA, Huson DH, Scölkopf B, Nordborg M, Rätsch G, Ecker JR, Weigel D. Common sequence polymorphisms shaping genetic diversity in Arabidopsis thaliana. Science. 2007;317(5836):338-42.
33. Wang L, Hao LX, Li X, Hu SL, Ge SX, Yu JK. SNP deserts of Asian cultivated rice: genomic regions under domestication. J Evol Biol. 2009;22(4):751-61.

34. Hu M, Yao SL, Cheng XH, Liu YY, Ma LX, Xiang Y, Huang JY, Tong CB, Liu SY. Genomic variation of spring, semi-winter and winter Brassica napus by highdepth DNA re-sequencing. Chin J Oil Crop Sci. 2018;40(4):469-78.

35. Chang FQ, Liu XM, Li YX, Jia GX, Ma J, Liu S, Zhou JQ. Analysis of low energy $\mathrm{N}^{+}$irradiation induced genome DNA variation of Arabidopsis thaliana. Sci China Ser C. 2003;33(2):117-24.

36. Springer NM, Ying K, Fu Y, Ji TM, Yeh CT, Jia Y, Wu W, Richmond T, Kitzman J, Rosenbaum H, Iniguez AL, Barbazuk WB, Jeddeloh JA, Nettleton D, Schnable PS. Maize inbreds exhibit high levels of copy number variation (CNV) and presence/absence variation (PAV) in genome content. PLoS Genet. 2009;5(11):e1000734.

37. Beló A, Beatty MK, Hondred D, Fengler KA, Li B, Rafalski A. Allelic genome structural variations in maize detected by array comparative genome hybridization. Theor Appl Genet. 2010;120(2):355.

\section{Publisher's Note}

Springer Nature remains neutral with regard to jurisdictional claims in published maps and institutional affiliations.
Ready to submit your research? Choose BMC and benefit from:

- fast, convenient online submission

- thorough peer review by experienced researchers in your field

- rapid publication on acceptance

- support for research data, including large and complex data types

- gold Open Access which fosters wider collaboration and increased citations

- maximum visibility for your research: over $100 \mathrm{M}$ website views per year

At BMC, research is always in progress.

Learn more biomedcentral.com/submissions 